\title{
THE MOTION OF COMET WESTPHAL IN 1852-1974
}

\author{
L. M. BELOUS
}

Institute of Theoretical Astronomy, Leningrad, U.S.S.R.

Two apparitions of Comet Westphal in 1852 and 1913 have been linked on the basis of 101 observations, the mean errors being equal to \pm 2 " 55 . The perturbations from nine major planets and the possible influence of nongravitational forces have been taken into account. The elements and the ephemeride for 1974 have been computed. 